\title{
QPCR: a tool for analysis of mitochondrial and nuclear DNA damage in ecotoxicology
}

\author{
Joel N. Meyer
}

Accepted: 17 December 2009/Published online: 5 January 2010

(C) The Author(s) 2009. This article is published with open access at Springerlink.com

\begin{abstract}
The quantitative PCR (QPCR) assay for DNA damage and repair has been used extensively in laboratory species. More recently, it has been adapted to ecological settings. The purpose of this article is to provide a detailed methodological guide that will facilitate its adaptation to additional species, highlight its potential for ecotoxicological and biomonitoring work, and critically review the strengths and limitations of this assay. Major strengths of the assay include very low (nanogram to picogram) amounts of input DNA; direct comparison of damage and repair in the nuclear and mitochondrial genomes, and different parts of the nuclear genome; detection of a wide range of types of DNA damage; very good reproducibility and quantification; applicability to properly preserved frozen samples; simultaneous monitoring of relative mitochondrial genome copy number; and easy adaptation to most species. Potential limitations include the limit of detection ( $\sim 1$ lesion per $10^{5}$ bases); the inability to distinguish different types of DNA damage; and the need to base quantification of damage on a control or reference sample. I suggest that the QPCR assay is particularly powerful for some ecotoxicological studies.
\end{abstract}

Keywords Mitochondrial DNA · DNA damage · DNA repair - Genotoxicity · Biomarker

Electronic supplementary material The online version of this article (doi:10.1007/s10646-009-0457-4) contains supplementary material, which is available to authorized users.

J. N. Meyer ( $\square)$

Nicholas School of the Environment, Duke University, Durham, Box 90328, NC 27708-0328, USA

e-mail: joel.meyer@duke.edu

\section{Introduction}

The quantitative polymerase chain reaction (QPCR) assay for DNA damage and DNA repair has been used to measure DNA damage and DNA repair for nearly 20 years (Kalinowski et al. 1992), mostly in laboratory model organisms. Primers and assay conditions have been described for Mus musculus, Rattus norvegicus, Drosophila melanogaster, Caenorhabditis elegans, Saccharomyces cerevisiae, Escherichia coli, Danio rerio, as well as for human samples (Hunter et al. submitted; Meyer et al. 2007; Santos et al. 2006). From an environmental perspective, it has been adapted to soybean (Cannon et al. 1995) and more recently the Atlantic killifish (Fundulus heteroclitus; Jung et al., 2009) and adenovirus (Eischeid et al. 2009), with other species anticipated (e.g., Daphnia magna; William Baldwin, personal communication). I refer to this assay as the "QPCR" assay since this is the historically used name. However, more recently, the term "long-amplicon quantitative PCR" ("LA-QPCR") has been used (Jung et al. 2009) to distinguish the assay from real-time PCR, sometimes also referred to as "quantitative PCR."

The QPCR assay works by amplifying large (typically $10-15 \mathrm{~kb})$ stretches of genomic DNA. Under quantitative conditions, any damage to the DNA that is able to stop or significantly inhibit the progression of the DNA polymerase used in the PCR reaction results in reduced amplification. Large amplicons are employed in order to improve the sensitivity of the assay by increasing the likelihood that a given amplification reaction will encounter damage. DNA damage detected by this assay has typically been referred to generically as "lesions" since many different types of damage (strand breaks, adducts, dimers, crosslinks, etc.) could inhibit the polymerase. All amplifications are performed under quantitative conditions (verified as described 
below in "QPCR development" parts 4 and 5), such that the amount of PCR product is dependent on the amount of starting template and the integrity of that template. To ensure that differential amplification reflects only damage, the reaction is carried out either using identical amounts of starting DNA template for all samples, or normalizing the amount of the large amplicons produced to the amount of small (usually 100-200 bases) amplicons produced. The small size of the normalization amplicons means that only a very high amount of DNA damage would inhibit their amplification. Finally, amplification of all samples is compared to amplification of control samples that are considered "undamaged" for the purposes of the assay. Any decrease in amplification from a sample compared to the control samples can be converted mathematically to a number of lesions per $\mathrm{kb}$ or $10 \mathrm{~kb}$ DNA, as described by Ayala-Torres et al. (2000), based on the assumption of random distribution of damage. Methods papers describing this assay have been published (Ayala-Torres et al. 2000; Hunter et al. submitted; Santos et al. 2006), but none have described the process of adapting the assay to new species, which is the goal of this technical note.

Figure 1 is a schematic that illustrates the large and small amplicons in a portion of a mitochondrial genome. Since the mitochondrial genomes of most organisms are $<20 \mathrm{~kb}$ in length, this assay typically amplifies the majority of that genome. The assay works in an analogous fashion in the nuclear genome. Figure 2, adapted from Ayala-Torres et al. (2000), is a schematic that illustrates how a QPCR experiment is carried out from exposure or environmental sampling (beginning) to calculation of lesion frequency (end). Additional descriptions of how the assay works are found in Van Houten et al. (2000), AyalaTorres et al. (2000), and Santos et al. (2006).

DNA repair can also be measured, by sampling DNA immediately after genotoxin exposure and at later time points. However, care must be taken to avoid potentially confounding effects of cell division or genome replication, as well as simultaneous damage and repair if the damaging agent is still present (such as would be the case for a chemical that is relatively long-lived in the organism).

In this technical note I provide a detailed guide to adapting the assay to new species, briefly and critically review major strengths and limitations of the QPCR assay, and summarize the potential of the assay for ecotoxicological research.

\section{Strengths and limitations of the QPCR assay}

\section{Strength \#1}

Very low (nanogram-picogram) amounts of DNA are required for analysis, since the assay is PCR-based.

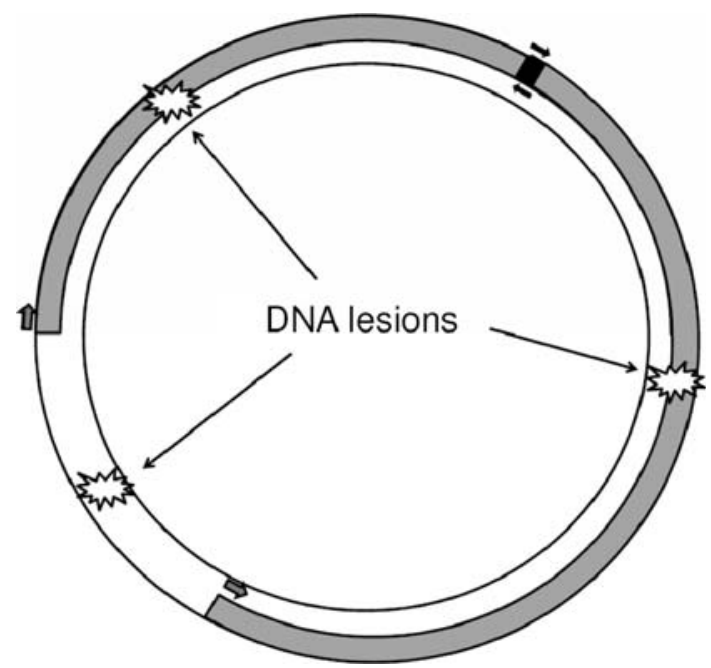

Fig. 1 Schematic rendering of the basis of the QPCR assay as it functions in the mitochondrial genome. The circular mitochondrial genome is represented as a white circle, the long amplicon (10-15 kb) is represented as a grey crescent that amplifies the majority of this genome, and the small amplicon ( $\sim 200$ bases $)$ is shaded black. Primers are represented as filled arrows. Lesions are represented as stars that would inhibit or block the progression of the DNA polymerase used in the PCR reaction, thus reducing the amplification of the long product under quantitative conditions. Amplification of the short product is not inhibited except by very high levels of damage: since the target is so small, in a large population of mitochondrial genomes, very few will have damage in the region amplified by the small product primers

QPCR reactions are typically performed on 5-15 ng template (input) DNA. Since the QPCR results are easier to interpret and more reliable if all reactions for all samples have a similar amount of input DNA, in most cases researchers start with larger (microgram) amounts of DNA that can be measured fluorometrically and diluted to equal concentrations. This is still a relatively low amount of DNA. Nonetheless, it is possible to use very small amounts of DNA. We have recently described analysis of DNA damage in individual larval and adult $C$. elegans (Boyd et al., 2010), which are composed of 500-1,000 cells or $\sim 50-150$ pg total genomic DNA. This input amount rivals that required by the COMET assay and is far less than most other genotoxicity assays, which typically require 10-50 $\mu$ g (e.g., Southern blot, HPLC, antibody-based, etc.).

\section{Strength \#2}

DNA damage and repair can be directly compared in the nuclear and mitochondrial genomes, since the QPCR assay is primer-based.

The mitochondrial genome is more sensitive than the nuclear genome to many genotoxins. For example, mitochondrial DNA is more vulnerable than nuclear DNA to exposure to oxidative damage of various sorts (Cover et al. 
Fig. 2 Schematic outline of how a QPCR experiment is carried out (adapted with permission from Ayala-Torres et al. 2000). The genotoxinexposed biological sample of interest (e.g., cells in a petri dish or a fish from a polluted site) is sampled, and total genomic DNA is extracted, quantified, and QPCR-amplified using the same amount of template genomic DNA input. Relative amplification of all samples is compared to amplification of control/reference samples to calculate DNA damage (lesion frequency)

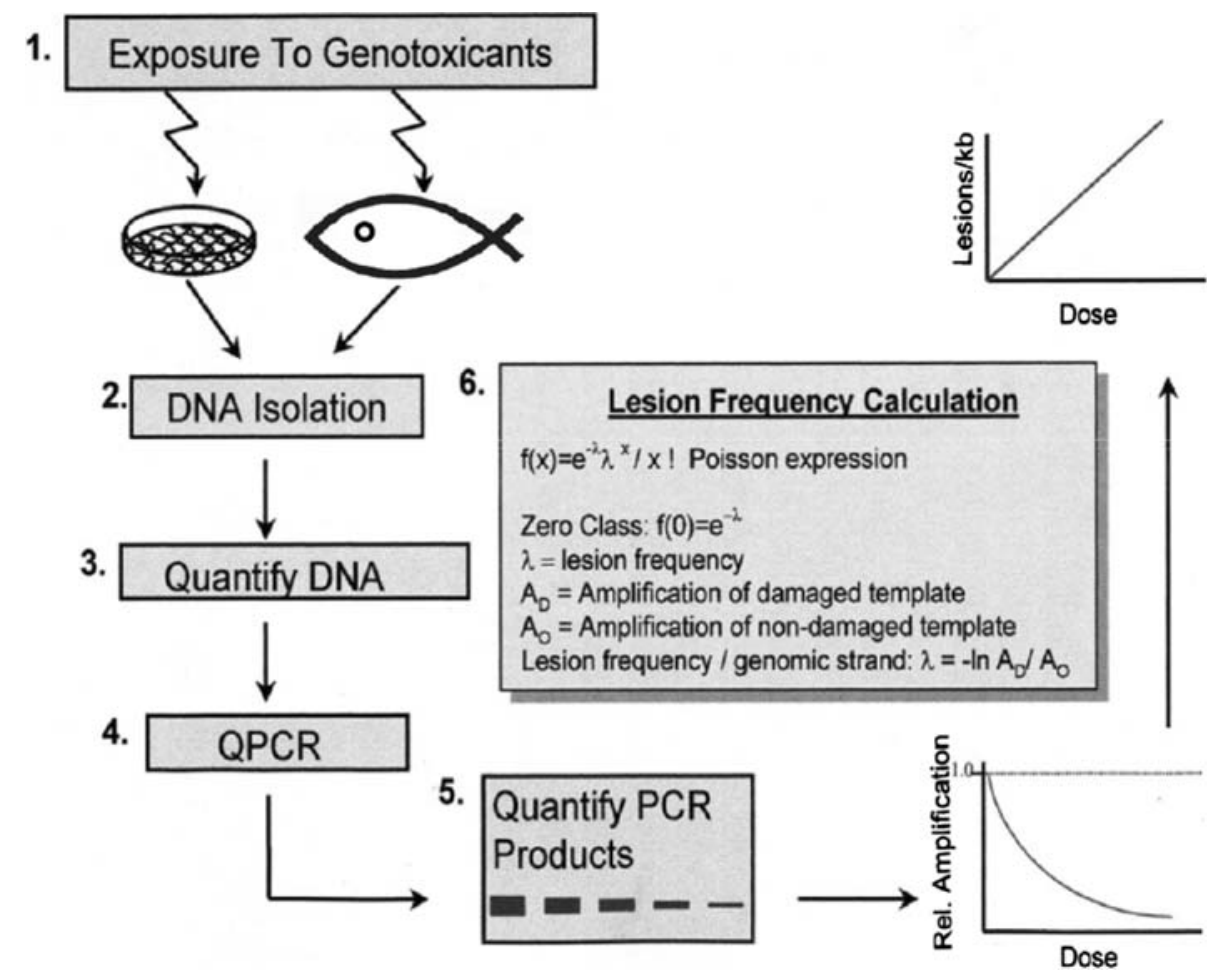

2005; Hollins et al. 2006; Salazar and Van Houten 1997; Santos et al. 2003; Yakes and Van Houten 1997), lipopolysaccharides (Suliman et al. 2003), benzo[a]pyrene and other polycyclic aromatic hydrocarbons (PAHs) (Allen and Coombs 1980; Backer and Weinstein 1980, 1982), photoactivated methylene blue (Anson et al. 2006), and aflatoxin (Niranjan et al. 1982). The increased sensitivity ranged in these reports from threefold (Yakes and Van Houten 1997) to several 100-fold (Allen and Coombs 1980). However, this is not true for all compounds. For example, cisplatin induced slightly more nDNA than mtDNA lesions (Van Houten et al. 2000). Some of these differences may also be larger in cell culture systems than in vivo; for example, PAH exposure seems to cause only several-fold more mitochondrial than nuclear DNA damage in vivo (Jung et al. 2009; Niranjan et al. 1982), not several hundred-fold as suggested by the in vitro studies cited above.

Repair of DNA damage is also different in the nuclear and mitochondrial genomes: not all nuclear DNA repair pathways exist in the mitochondria. In most species, it appears that base excision repair, and possibly mismatch repair and recombinational repair are present in the mitochondria, but nucleotide excision repair (NER) is not (Anson et al. 2006; Bogenhagen 1999; Croteau et al. 1999; Kraytsberg et al. 2004; LeDoux et al. 1999; Marcelino and Thilly 1999; Sawyer and Van Houten 1999). This is significant because NER is responsible for the repair of a large number of environmentally significant lesions (Hanawalt 2002; Hoeijmakers 2001). For example, NER removes and replaces bulky adducts caused by benzo $[a]$ pyrene diol epoxide and other metabolites of polycyclic aromatic hydrocarbons and aflatoxin, as well as ultraviolet lightinduced photodimers (Friedberg et al. 2006c).

Since the QPCR assay is primer-based, differential treatment in order to separate mitochondrial from nuclear DNA is not required. This is critical since such differential treatments can lead to artifactual damage (Anson et al. 2000; Beckman and Ames 1999).

\section{Strength \#3}

The QPCR assay permits simultaneous monitoring of alterations in relative mitochondrial genome copy number.

Mitochondrial genome copy number can vary in response to a variety of stressors (Lee and Wei 2005), as well as in different tissues, developmental stages, etc. Comparison between samples of the degree of amplification of the small mitochondrial product allows quantification of any change in the relative copy number of the mitochondrial genome compared to the nuclear genome.

\section{Strength \#4}

DNA damage and repair can be directly compared in different parts of the nuclear genome.

By using primers that amplify different portions of the nuclear genome (Meyer et al. 2007; Van Houten et al. 2000), it is also possible to test whether different parts of 
the nuclear genome are more damaged or differentially repaired. For example, more transcriptionally active portions of the nuclear genome that are less packaged may be more exposed to genotoxins (Friedberg et al. 2006b), and more transcriptionally active regions of the genome may be more quickly repaired (Hanawalt and Spivak 2008).

\section{Strength \#5}

Since the QPCR assay detects any damage that inhibits polymerase progress, a wide range of types of DNA damage can be detected.

Thus, it is not necessary to know ahead of time what lesion type(s) are most important. Nor are multiple analyses of different types of damage required to quantify a "total" (summed) level of damage. Finally, it is unnecessary to introduce damage-specific breaks in DNA before measuring the damage (as required in some cases for the COMET and Southern blot assays).

\section{Strength \#6}

Because the QPCR assay is primer-based, it can easily be adapted to any species for which significant genomic DNA sequence exists or can be obtained.

The database of such genomic sequence is expanding very rapidly, and is already very large for mitochondrial sequences. Sequence data can be obtained from general multi-organismal databases such as GenBank (http://www. ncbi.nlm.nih.gov/Genbank/) or, when available, organismspecific genome sequence databases (e.g., http://wfleabase. org/database/ for Daphnia). The ability to obtain de novo sequence information has also increased dramatically.

\section{Strength \#7}

Since the QPCR assay is primer-based, the presence of DNA from other species in a sample will not bias measurements.

This assumption can and should be tested empirically.

\section{Strength \#8}

The QPCR assay can be performed on properly preserved, frozen samples.

In particular, tissue samples or organisms can be stored at $-80^{\circ} \mathrm{C}$ for months or years.

\section{Strength \#9}

The QPCR assay yields highly reproducible and quantifiable results.
Ultraviolet (UV) C radiation-induced DNA damage has been most studied by different researchers using QPCR, and results have been quite reproducible. Eischeid et al. (2009) graphed UV-induced DNA damage measured in several types of samples and by different researchers. For the cell lines, bacteria and virus samples examined, the damage induced fit one dose-response curve; slightly more damage was induced in naked DNA, as expected given the lack of any shielding. There are relatively few assay parameters that will lead to large inter-laboratory differences with the QPCR assay. This is not the case for some other common biomonitoring genotoxicity assays, such as the COMET assay (Frenzilli et al. 2009; Lee and Steinert 2003; Valverde and Rojas 2009). Furthermore, it is possible to define an actual number of lesions per kb genomic DNA (Ayala-Torres et al. 2000), which is also difficult with the COMET assay (Friedberg et al. 2006a).

\section{Limitation \#1}

The limit of detection of the QPCR assay is $\sim 1$ lesion per $10^{5}$ bases.

This limit of detection permits good sensitivity, but is higher than that of at least one other DNA damage assay, the COMET assay. For example, the COMET is reported to detect approximately UVC-induced DNA damage at $0.2 \mathrm{~J} / \mathrm{m}^{2}$ in HeLa cells (Collins et al. 1997), which corresponds to >tenfold more sensitivity than the QPCR assay.

\section{Limitation \#2}

The inability to distinguish different types of DNA damage may be a limitation for some studies.

While this feature is in some ways advantageous (Strength \#5), it can be problematic if the goal is to study a specific type of damage. One solution is to follow up the QPCR assay, when warranted, with a more specific assay.

Relatedly, the degree to which different types of lesions inhibit the commercial DNA polymerase preparations used for the assay is not fully characterized (Santos et al. 2006; Sikorsky et al. 2004). However, the assay has been used to successfully detect DNA damage caused by dozens of different genotoxins causing a wide range of damage including bulky DNA adducts, alkylating agents, oxidative damage, and strand breaks (Ayala-Torres et al. 2000).

\section{Limitation \#3}

Damage is measured by comparing amplification of "test" samples to amplification from control (or reference) samples.

While the lesion numbers generated are absolute and not relative, they are calculated from a baseline that is defined 
as zero; i.e., the amplification of the control samples is considered to represent amplification of lesion-free DNA. This concern is likely to be less important in a laboratory setting where background lesion frequencies are unlikely to be detectable by this assay, but is potentially an issue in the context of ecotoxicological studies. This can be addressed by careful choice and/or testing of reference sites.

\section{Limitation \#4}

The QPCR assay is not cell-specific.

The QPCR assay has so far not been carried out on single cells, but rather on batches of cells. Thus, potential cell-to-cell variability is not measured. The assay itself is highly reproducible, assuming training in molecular biology and adequate quality control in sample processing (see below).

\section{Potential of the QPCR assay for biomonitoring and mechanistic ecotoxicology}

Genotoxicity is an important area of ecotoxicological research from both mechanistic and biomonitoring perspectives (Anderson et al. 1994; Depledge 1998; Hinton et al. 2005; Jha 2004; Wirgin and Waldman 1998). The QPCR assay has the potential to contribute to both. It has already been used extensively for mechanistic studies, generally in the context of human health (reviewed in (Santos et al. 2006). It has also been applied to a limited degree for biomonitoring in humans (Haugen et al. in press) and the ecological sentinel species $F$. heteroclitus (Jung et al. 2009). A complete comparative review of different genotoxicity biomarkers is outside of the scope of this manuscript, and different assays clearly have different strengths and limitations. The COMET assay is one of the most frequently used assays for DNA damage in biomarker studies (Dusinska and Collins 2008), and so I have drawn some comparisons to the COMET assay. I highlight below how the strengths of the QPCR assay (detailed above) contribute to its potential utility for ecotoxicological studies.

From a biomonitoring perspective, the QPCR assay offers the ability to measure DNA damage in very small samples, because of the low amount of input DNA required. This might allow examination of damage in very small species (e.g., nematodes, water fleas, etc.) or biopsies or blood samples from larger individuals. Since most nonmammalian vertebrates have nucleated red blood cells, there is an excellent potential for such studies in many wildlife species. Another reason that the QPCR assay may be particularly advantageous for wildlife biomonitoring is its utility for the measurement of mitochondrial DNA damage. As discussed above, mtDNA is especially vulnerable to some types of genotoxins, and the types of damage that can be repaired are limited in the mitochondrial compared to the nuclear genome. Thus, mtDNA damage detected by the QPCR assay has been previously suggested as a biomarker of oxidative DNA damage (Mandavilli et al. 2002) and PAH exposure (Jung et al. 2009). Finally, as also previously indicated (Jung et al. 2009), the QPCR assay may be particularly easy to adapt to many non-laboratory species for which molecular tools are otherwise lacking, because of the widespread availability of mitochondrial genome sequences obtained for phylogenetic and evolutionary studies. The same should be true of very highly-conserved nuclear-encoded genes (e.g., ribosomal RNA genes).

From a mechanistic perspective, the QPCR assay is frequently utilized for analyses comparing damage and repair in mitochondrial versus nuclear DNA. The only other assay that offers this capacity without differential extraction is the Southern blot assay (Anson et al. 2006), which has a much higher requirement for input DNA and requires the introduction of DNA damage-dependent strand breaks. The ability to measure damage and repair in different regions of the nuclear genome is also powerful, as discussed above. Finally, from a specifically ecotoxicological mechanistic perspective, the relative importance of the mitochondrial genome as a target of genotoxins is almost entirely unexplored outside of the realm of human health, where it is increasingly understood to play a critical role (Penta et al. 2001; Van Houten et al. 2006; Wallace 2005; Weissman et al. 2007). We simply do not know how important mtDNA damage may or may not be in other species, and the QPCR assay is the best tool to use to address this question. Further reason to suspect that looking for mtDNA damage would be worthwhile is the observation of elevated mtDNA heteroplasmy in both human (Forster et al. 2002) and wildlife (Matson et al. 2006) populations exposed to genotoxins.

A final important consideration is logistical. Equipment required is for the most part standard for a molecular biology laboratory and relatively inexpensive, with the exception of high quality thermocyclers and a fluorescence plate reader. A thermocycler with gradient capacity facilitates PCR optimization but is not essential. Processing (from DNA extraction to data analysis) costs are $<\$ 10$ / sample with two PCR replicates and analyzing both nuclear and mitochondrial genomes, if DNA is sampled via sample lysis. The cost rises to $\sim \$ 15 /$ sample if larger samples are homogenized and DNA extracted. Different extraction options are described below. With sample lysis, processing can be carried out in 1-2 days; $4-8 \mathrm{~h}$ of processing time is added when DNA is extracted from larger samples, depending on the number of samples. 


\section{Guide for adaptation to additional species}

Adaptation of the QPCR assay to a new species is fairly straightforward if significant genomic DNA sequence is available or can be obtained. Two protocols will need to be optimized: extraction of high molecular weight, high quality DNA, and the QPCR assay itself. The following guide is designed to orient a person already familiar with basic molecular biology techniques (e.g. DNA extraction, gel electrophoresis, primer design, and PCR).

\section{DNA extraction}

Since the QPCR assay involves amplification of large $(>10 \mathrm{~kb})$ products, it is critical that it be carried out on high molecular weight, high quality DNA. For larger organisms from which soft tissues are sampled, DNA may be extracted via homogenization as previously described (Santos et al. 2006) for laboratory organisms. However, for organisms with barriers such as cuticles, additional extraction steps may be required. For example, DNA is best extracted from batches of $C$. elegans by first freezing the batch and then grinding it in liquid nitrogen. Phenolbased extraction is not recommended, as it can result in oxidation of DNA (Helbock et al. 1998). A protocol for extraction of high molecular weight DNA from C. elegans, including extraction kit suggestions and methods for evaluating the integrity of the extracted DNA, is presented as Supplemental data file 1.

In some cases, it is possible to carry out QPCR directly on proteolytically digested lysates of very small samples. For example, we found that it was possible to measure DNA damage in a single nematode after lysis/digestion (Boyd et al. 2010). The protocol used for that procedure is presented as Supplemental data file 2 (see also Boyd et al. 2010). However, there is too little DNA present in such samples to be analyzed for integrity by gel electrophoresis. Therefore, before accepting such an approach, it is critical to compare damage levels as measured in the same sample using a traditional DNA extraction approach (e.g., liquid nitrogen grinding followed by column separation) with those obtained using novel extraction methods.

\section{QPCR development}

Detailed protocols for how to carry out the QPCR assay have been published (Hunter et al. submitted; Meyer et al. 2007; Santos et al. 2006) and will not be repeated here. However, those protocols should be read prior to carrying out the following steps. They include guidance on quantification of template DNA using picogreen dye and a plate reader, typical PCR reaction conditions, choice of DNA polymerase, quantification of product using picogreen and a plate reader, and primer sequences. The requirement to not open PCR reactions in the same room where DNA is extracted and PCR reactions are set up is critical!

Following are the steps involved in adapting the QPCR assay to a new species:

1. Determine what gene or genomic area(s) you wish to amplify in the QPCR reaction, and design primers for short and long products for those targets. Primers for one long (10-15 kb) and one short ( $\sim 200 \mathrm{bp}$ ) product from each genome will be sufficient for most researchers whose objective is to develop a tool for detecting damage in wildlife samples. Multiple targets are probably not important if the intended use of the assay is as a biomarker, since very dramatic differences in damage in different regions of the nuclear genome have not been reported with this assay (although this could conceivably change in the future). Similarly, it is not especially important from the biomarker perspective which portion of the nuclear genome is amplified (coding, noncoding, etc.): any region will do and is assumed to be reasonably representative of the rest of the nuclear genome. However, researchers interested in detecting possible differential damage or repair in different regions of the genome (e.g., transcribed vs. nontranscribed, as described in Strength \#4) will want to design primers for multiple targets. In addition, as noted earlier, it is important to separately assess damage to the nuclear and mitochondrial genomes, since damage and repair are often quite different. Finally, highly repetitive regions of the nuclear genome should be avoided as they will make the amplification of a highly specific product difficult.

The primers can probably be designed with any software, but should allow for a very high annealing temperature (typically near $70^{\circ} \mathrm{C}$ for large products, and $65^{\circ} \mathrm{C}$ for small products) in order to generate unique PCR products (i.e., preclude nonspecific annealing). Other standard primer design considerations also apply (avoid primer-dimers, self-annealing, etc.). To save time, order at least three pairs of primers for each product desired and test all combinations. A Word file that illustrates the primer design process in more detail using Primer3 software (Rosen and Skaletsky 2000) is presented as Supplemental data file 3 , and more details and recommendations for primer design are presented in Supplemental data file 4.

If template DNA will always be extracted by a batch procedure, it is possible to dispense with designing primers for a short nuclear product. Since nuclear DNA normally constitutes $>99 \%$ of total cellular DNA, template DNA quantified with picogreen (Santos et al. 2006) can be diluted to equal concentrations 
for all samples so that these reactions will not require small nuclear product-derived normalization.

2. Pick the best primer combinations for each target. The QPCR reactions should be robust and specific: look for a product that is very well-amplified and unique or close to unique on an ethidium bromide-stained gel. One or a few very minor off-target bands can usually be eliminated by optimization (step 3, below). An example of primer testing including a sample gel is provided as part of Supplemental data file 4. The identity of the amplified product can initially be assumed to be correct based on correct product size, but identity should be confirmed via sequencing or restriction digest followed by electrophoresis of the products, to confirm that products of the expected size are generated.

3. Optimize critical reaction conditions for each $Q P C R$ reaction. Having chosen the best pair of primers for each target, test a range of magnesium concentrations and annealing temperatures in order to generate a unique product that amplifies well. Detailed suggestions are provided in Supplemental data file 4.

4. Carry out a cycle test to determine the cycle range over which a given amount of input template yields a log-linear increase. The "right" amount of input template will depend somewhat on the source of DNA. For batch-extracted DNA, 10-15 ng is typically used. It may also be possible to use a biological unit (e.g., one nematode), provided that the biology of the sample is such that the DNA amount will be very similar from one unit to the next. Example gels for cycle tests are provided in Supplemental data file 4.

5. Carry out template tests to ascertain that the assay is performing quantitatively. If the QPCR is performing quantitatively, reducing to $50 \%$ or increasing to $200 \%$ the amount of template DNA input should lead to a two-fold decrease or increase in PCR product output. Examples are provided in Supplemental data file 4.

6. Test with positive control. It should be possible to generate DNA damage and detect that damage using a model genotoxin. One of the simplest is UVC, although other genotoxins may be fine. UVC is useful because it is easy to generate (e.g., a UV crosslinker or UV lamp with meter) and expected dose-responses are well-defined. For example, DNA damage generated by $5 \mathrm{~J} / \mathrm{m}^{2}$ UVC in "naked" DNA (extracted DNA in a small volume of Tris-EDTA buffer) should be in the range of 1.5-2 lesions/10 kb (Eischeid et al. 2009). A sample Excel spreadsheet with simulated fluorescence data and conversions to lesions/10 $\mathrm{kb}$ is provided as Supplemental data file 5, with additional details regarding that spreadsheet provided in Supplemental data file 4. Supplemental data file 5 also points out how to use this assay to measure relative mitochondrial genome copy number changes.

As a final note, this assay has historically been carried out in a cycle-optimized (endpoint) fashion rather than via real-time PCR. This is in part because of the challenge of amplifying large products using real-time PCR. However, a real-time version of this assay was recently described (Edwards 2009), so it may be worthwhile to develop this assay for a real-time thermocycler.

Acknowledgment This work was supported by NIH P42 ES10356.

Open Access This article is distributed under the terms of the Creative Commons Attribution Noncommercial License which permits any noncommercial use, distribution, and reproduction in any medium, provided the original author(s) and source are credited.

\section{References}

Allen JA, Coombs MM (1980) Covalent binding of polycyclic aromatic compounds to mitochondrial and nuclear DNA. Nature. 287:244-245

Anderson S et al (1994) Genetic and molecular ecotoxicology: a research framework. Environ Health Perspect 102(Suppl12):3-8

Anson RM et al (2000) Mitochondrial endogenous oxidative damage has been overestimated. FASEB J 14:355-360

Anson RM et al (2006) Gene-specific and mitochondrial repair of oxidative DNA damage. Methods Mol Biol 314:155-181

Ayala-Torres S et al (2000) Analysis of gene-specific DNA damage and repair using quantitative polymerase chain reaction. Methods 22:135-147

Backer JM, Weinstein IB (1980) Mitochondrial DNA is a major cellular target for a dihydrodiol-epoxide derivative of benzo[a]pyrene. Science. 209:297-299

Backer JM, Weinstein IB (1982) Interaction of benzo(a)pyrene and its dihydrodiol-epoxide derivative with nuclear and mitochondrial DNA in C3H10T 1/2 cell cultures. Cancer Res 42:2764-2769

Beckman KB, Ames BN (1999) Endogenous oxidative damage of mtDNA. Mutat Res 424:51-58

Bogenhagen DF (1999) Repair of mtDNA in vertebrates. Am J Hum Genet 64:1276-1281

Boyd WA et al (2010) Nucleotide excision repair is not detectably inducible, but is required for normal lifespan and growth, in genotoxin-stressed adult Caenorhabditis elegans. Mutat Res/ Fundam Mol Mech Mutagen 683:57-67

Cannon GC et al (1995) Repair mechanisms of UV-induced DNA damage in soybean chloroplasts. Plant Mol Biol 29:1267-1277

Collins AR et al (1997) The comet assay: what can it really tell us? Mutat Res 375:183-193

Cover C et al (2005) Peroxynitrite-induced mitochondrial and endonuclease-mediated nuclear DNA damage in acetaminophen hepatotoxicity. J Pharmacol Exp Ther 315:879-887

Croteau DL et al (1999) Mitochondrial DNA repair pathways. Mutat Res DNA Repair 434:137-148

Depledge MH (1998) The ecotoxicological significance of genotoxicity in marine invertebrates. Mutat Res 399:109-122

Dusinska M, Collins AR (2008) The comet assay in human biomonitoring: gene-environment interactions. Mutagen 23: $191-205$ 
Edwards JG (2009) Quantification of mitochondrial DNA (mtDNA) damage and error rates by real-time QPCR. Mitochondrion 9:31-35

Eischeid AC et al (2009) UV disinfection of adenoviruses: molecular indications of DNA damage efficiency. Appl Environ Microbiol $75: 23-28$

Forster L et al (2002) Natural radioactivity and human mitochondrial DNA mutations. Proc Natl Acad Sci USA 99:13950-13954

Frenzilli G et al (2009) The Comet assay for the evaluation of genotoxic impact in aquatic environments. Mutat Res 681:80-92

Friedberg EC et al (2006a) DNA repair and mutagenesis. ASM Press, Washington, DC, p 273

Friedberg EC et al (2006b) DNA repair and mutagenesis. ASM Press, Washington, DC, pp 48-49

Friedberg EC et al (2006c) DNA Repair and Mutagenesis. ASM Press, Washington, DC, p 29

Hanawalt PC (2002) Subpathways of nucleotide excision repair and their regulation. Oncogene 21:8949-8956

Hanawalt PC, Spivak G (2008) Transcription-coupled DNA repair: two decades of progress and surprises. Nat Rev Mol Cell Biol 9:958-970

Haugen AC et al (in press) Altered gene expression and DNA damage in peripheral blood cells from Friedreich's ataxia patients: Cellular Model of Pathology. PLOS Genetics

Helbock HJ et al (1998) DNA oxidation matters: the HPLCelectrochemical detection assay of 8-oxo-deoxyguanosine and 8-oxo-guanine. Proc Natl Acad Sci USA 95:288-293

Hinton DE et al (2005) Resolving mechanisms of toxicity while pursuing ecotoxicological relevance? Mar Pollut Bull 51:635648

Hoeijmakers JH (2001) Genome maintenance mechanisms for preventing cancer. Nature 411:366-374

Hollins DL et al (2006) Glutathione regulates susceptibility to oxidant-induced mitochondrial DNA damage in human lymphocytes. Free Radic Biol Med 40:1220-1226

Hunter S et al (submitted) The QPCR assay for analysis of mitochondrial DNA damage, repair, and relative copy number. Methods

Jha AN (2004) Genotoxicological studies in aquatic organisms: an overview. Mutat Res 552:1-17

Jung D et al (2009) The long amplicon quantitative PCR for DNA damage assay as a sensitive method of assessing DNA damage in the environmental model, Atlantic killifish (Fundulus heteroclitus). Comp Biochem Physiol C Toxicol Pharmacol 149:182-186

Kalinowski DP et al (1992) Analysis of DNA damage and repair in murine leukemia L1210 cells using a quantitative polymerase chain reaction assay. Nucleic Acids Res 20:3485-3494

Kraytsberg Y et al (2004) Recombination of human mitochondrial DNA. Science 304:981

LeDoux SP et al (1999) Repair of alkylation and oxidative damage in mitochondrial DNA. Mutat Res 434:149-159

Lee RF, Steinert S (2003) Use of the single cell gel electrophoresis/ comet assay for detecting DNA damage in aquatic (marine and freshwater) animals. Mutat Res 544:43-64

Lee HC, Wei YH (2005) Mitochondrial biogenesis and mitochondrial DNA maintenance of mammalian cells under oxidative stress. Int J Biochem Cell Biol 37:822-834
Mandavilli BS et al (2002) Mitochondrial DNA repair and aging. Mutat Res 509:127-151

Marcelino LA, Thilly WG (1999) Mitochondrial mutagenesis in human cells and tissues. Mutat Res 434:177-203

Matson CW et al (2006) Evolutionary toxicology: population-level effects of chronic contaminant exposure on the marsh frogs (Rana ridibunda) of Azerbaijan. Environ Health Perspect 114:547-552

Meyer JN et al (2007) Decline of nucleotide excision repair capacity in aging Caenorhabditis elegans. Genome Biol 8:R70

Niranjan BG et al (1982) Preferential attack of mitochondrial DNA by aflatoxin B1 during hepatocarcinogenesis. Science 215:73-75

Penta JS et al (2001) Mitochondrial DNA in human malignancy. Mutat Res 488:119-133

Rosen S, Skaletsky HJ (2000) Primer3 on the WWW for general users and for biologist programmers. In: Krawetz S, Misener S (eds) Bioinformatics methods and protocols: methods in molecular biology. Humana Press, Totowa, NJ, pp 365-386

Salazar JJ, Van Houten B (1997) Preferential mitochondrial DNA injury caused by glucose oxidase as a steady generator of hydrogen peroxide in human fibroblasts. Mutat Res 385:139-149

Santos JH et al (2003) Cell sorting experiments link persistent mitochondrial DNA damage with loss of mitochondrial membrane potential and apoptotic cell death. J Biol Chem 278:17281734

Santos JH et al (2006) Quantitative PCR-based measurement of nuclear and mitochondrial DNA damage and repair in mammalian cells. Methods Mol Biol 314:183-199

Sawyer DE, Van Houten B (1999) Repair of DNA damage in mitochondria. Mutat Res 434:161-176

Sikorsky JA et al (2004) Effect of DNA damage on PCR amplification efficiency with the relative threshold cycle method. Biochem Biophys Res Commun 323:823-830

Suliman HB et al (2003) Postlipopolysaccharide oxidative damage of mitochondrial DNA. Am J Respir Crit Care Med 167:570-579

Valverde M, Rojas E (2009) Environmental and occupational biomonitoring using the Comet assay. Mutat Res 681:93-109

Van Houten B et al (2000) Measuring gene-specific nucleotide excision repair in human cells using quantitative amplification of long targets from nanogram quantities of DNA. Mutat Res 460:91-94

Van Houten B et al (2006) Role of mitochondrial DNA in toxic responses to oxidative stress. DNA Repair (Amst) 5:145-152

Wallace DC (2005) A mitochondrial paradigm of metabolic and degenerative diseases, aging, and cancer: a dawn for evolutionary medicine. Annu Rev Genet 39:359-407

Weissman L et al (2007) DNA repair, mitochondria, and neurodegeneration. Neuroscience 145:1318-1329

Wirgin I, Waldman JR (1998) Altered gene expression and genetic damage in North American fish populations. Mutat Res 399:193-219

Yakes FM, Van Houten B (1997) Mitochondrial DNA damage is more extensive and persists longer than nuclear DNA damage in human cells following oxidative stress. Proc Natl Acad Sci USA 94:514-519 